\title{
Nursing care of patients in the prone position
}

Birte Baktoft RN, CCN, Clinical Nurse Specialist, Intensive Care Unit, Randers County Hospital, Denmark

Birte Baktoft, Intensive Care Unit, Randers County Hospital, Skovlyvej 1, DK-8900 Randers, Denmark.

e-mail: birte.baktoft@teliamail.dk

\section{INTRODUCTION}

Acute respiratory distress syndrome (ARDS) is a well-known disease; a dreaded complication of patients in intensive care units (ICUs) all over the world. It was described in the literature, as adult respiratory distress syndrome, for the first time almost 35 years ago (Ashbaugh et al., 1967). A few years later, the prone position was introduced for the treatment of ARDS (Bryan, 1974). Many clinical studies have confirmed the positive effect of prone positioning on oxygenation in patients, describing it as having a 'dramatic effect on oxygenation in patients with severe acute lung insufficiency’ (Mure et al., 1997). However, a large Italian study presented by Gattinoni, at the 21st International Symposium on Intensive Care and Emergency Medicine held in Brussels, Belgium, in 2001, showed that the survival of ARDS patients was not improved by turning the patients into the prone position.

It is not the intention of this article to discuss the pros and cons of prone ventilation. This article will focus on the clinical nursing care of such patients.

\section{BACKGROUND}

In my ICU, at Randers County Hospital, Denmark, we turned our first patient into the prone position four years ago. Previously, one of the ICU doctors and I went to the Rudolph Virchov Klinikum, a large university hospital in Berlin, Germany. One of their ICUs specialised in the treatment of ARDS and caring for patients in the prone position. We stayed there for four rewarding days. On our return to Denmark, we trained the whole staff (doctors and nurses) and introduced prone position ventilation of patients into our ICU. Through experience, I would like to emphasise the importance of making thorough preparations before introducing such a complex nursing task into your ICU.

Before developing nursing guidelines, we investigated the nursing literature. Many articles described the physiological effects of turning the patient prone; how it affects the haemodynamics, oxygenation and so on. However, very few articles dealt with nursing care, such as how to avoid complications during the turning procedure, preventing pressure sores, or how to perform endotracheal suction or feed the patient. We gathered evidence from a number of sources and took the best from a few articles, from our study visit to Berlin, and from our own experiences. Finally we formulated our own set of nursing guidelines, of which our key objective is that:
No patient should develop complications in connection with either the turning procedure or the prone position.

The remainder of this article describes the nursing procedures and care of patients ventilated in the prone position. It is based on our own guidelines and our experience of developing best practice.

\section{KEY NURSING POINTS FOR THE PRONE POSITION Indications for the prone position}

The patients that we turn into the prone position are predominantly those with ARDS. We also turn patients with severe pneumonia and/or other conditions, in whom oxygenation is a major problem.

In our unit, the indication for turning a patient is: ventilator settings with oxygen support $>60 \%\left(\mathrm{FiO}_{2}>0.6\right)$ to keep the patient's haemoglobin oxygen saturation $\left(\mathrm{SaO}_{2)} \geq 90 \%\right.$. However, before we turn a patient we perform a chest X-ray to exclude pneumothorax. The potential for any other technical or mechanical exclusion is also discussed.

It is very important that complications do not occur during the turn. In order to help ensure a safe and smooth process we take several precautions (Table 1 ).

\section{Table 1. Precautions to be taken before turning a patient into the prone position}

Inform the relatives if they are present in the unit

- Five people must be present. One must be a doctor, who is responsible for the endotracheal tube (Stocker et al., 1996). The others can be nurses (or possibly assistant health workers)

- Select one - and only one - person to be responsible for directing the turning procedure (usually the doctor standing at the head of the bed)

- Make sure that the patient is well-sedated

Make sure that the endotracheal tube is anchored securely

Protect eyes with ointment and eye pads

- Make sure that all equipment and medicine for potential resuscitation are within reach

- Disconnect and remove all the equipment that the patient can do without

- Adjust the remaining infusion lines and monitor leads to prevent kinking and disconnection

- Just before turning, remove the electrodes from the patient's chest 


\section{Turning procedure}

The direction of the turn must always be towards the ventilator (Vollman, 1997).

We use two different methods for turning the patient.

\section{Method One}

- A sheet is placed over the patient.

- The bottom sheet is loosened, and both sheets are rolled together - along the sides of the patient.

- Two nurses pull the patient - who now is wrapped into the sheets - to the edge of the bed, and slowly turn him over.

- Two nurses on the other side carefully position the patient and unroll the sheets.

- The clean sheet is now underneath the patient.

\section{Method Two}

- Two nurses pull the patient to the edge of the bed - away from the ventilator.

- The arm nearest to the ventilator is placed under the back of the patient.

- The opposite leg is placed across the other leg.

- The patient is then slowly turned around his own axis into the prone position.

No matter which method we use, the doctor is continuously holding the endotracheal tube. Immediately after turning the patient, the electrodes and the saturation probe are replaced. Finally, pillows are placed under the patient's chest and pelvis to avoid abdominal pressure from the mattress. It is also important to ensure that the patient is not lying on an infusion line, drain, or catheter, etc. The patient's face must point directly to the mattress and should never be turned from one side to another. This is to prevent compression of blood vessels in the neck (Mure et al., 1997). A special pillow supports the head. We use two different types of pillow; both types are made from a special material designed to prevent pressure sore development (Figures 1 and 2).

Our patients are left in prone position for an average of 12 hours, depending on their response to the treatment. A few patients are turned several times. One patient was turned 11 times before the ARDS was under control. When a patient is prone, it is important to keep an eye - or, I should say a hand on the abdomen to ensure free abdominal wall motion. It should be easy to get a hand between the mattress and the abdomen. If not, more pillows should be placed under the chest and pelvis.

\section{Avoiding pressure sores}

Pressure sores are a common complication. The forehead, chin, pelvis and knees are the most vulnerable areas. So too, are the corner of the mouth (from the endotracheal tube) and the tip of the nose (from the feeding tube). The patients are lying in the prone position for a long time, and many have universal oedema, which increases the risk of pressure sore development. However, a large Swedish study showed that, in the $76 \%$ of ICUs that performed prone position ventilation, only $16 \%$ of patients developed pressure sores (Gustavsson and Jonsson, 1998).

It is still being debated which is the best type of mattress and pillow for prone ventilation. Gustavsson and Jonsson (1998) suggested that the actual choice of pillows and mattresses is a minor factor only in the development of pressure sores. What is

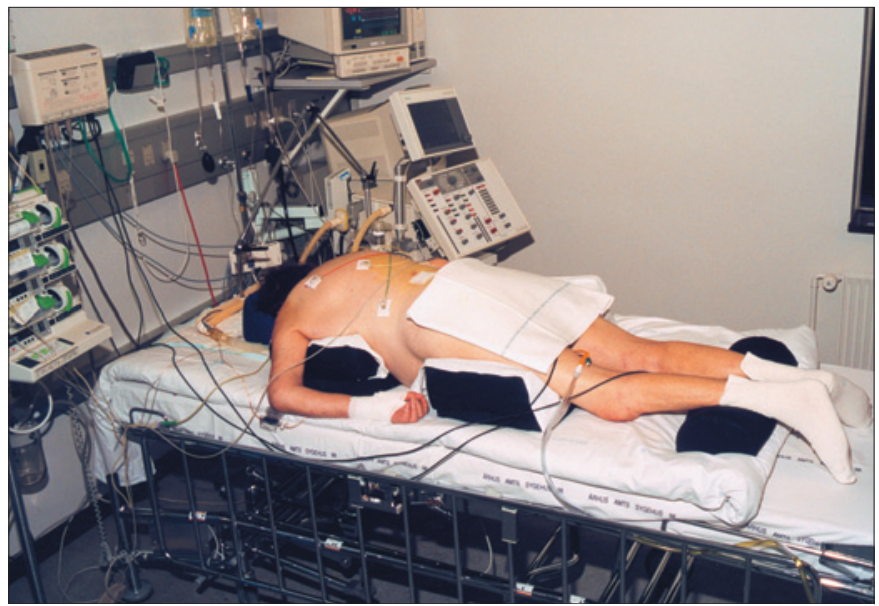

Figure 1.

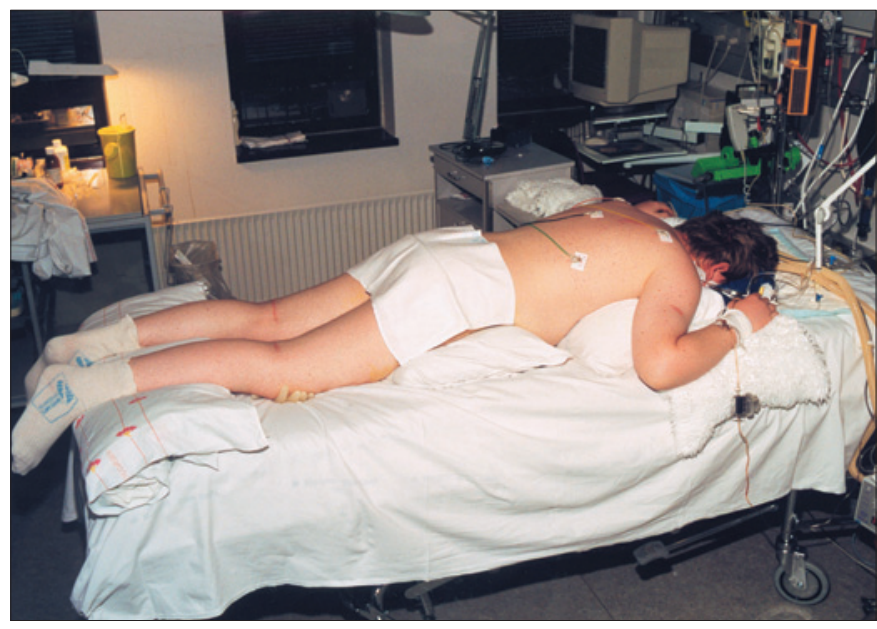

Figure 2.

important is that they are made of anti-pressure sore materials. One should not use ordinary duvets, pillows or blankets to prop up the patient (Gustavsson and Jonsson, 1998). This result is in line with our experience. As mentioned earlier, we use two different types of pillows to support the body, and there seems to be no difference in the incidence of pressure sores.

To support the face, we now use a pillow developed by a medical company in Denmark, in collaboration with an ICU in Randers and an ICU in Kolding. The pillow does not prevent the oedema of the face, but it reduces markedly the incidence of pressure sores and marking. To avoid pressure sores in the face, two nurses carefully position the patient's head to ensure that the endotracheal tube and the feeding tube do not press against the mouth and the tip of the nose.

At least every second hour, we change the position of the arms and legs to move the pressure points. We constantly ensure that the patient not is lying on infusion lines, etc. In males, it is particularly important to ensure there is no pressure on the genital area.

\section{Physiotherapy}

A few rare complications of the prone ventilation position are described, such as calcification of shoulders (long-term positioning, e.g. 26 days) and nipple necrosis (Willems et al., 1998).

Having a heavily sedated patient lying in the same position for many hours requires frequent exercise of all extremities to avoid stiffness and contraction. Our physiotherapist is very much aware of this. She made a series of photographs to illu- 
strate the correct procedures for exercising and positioning the joints, which focuses on the shoulders, arms, legs, feet and the fingers (Figures 3, 4 and 5). These exercises are now part of our protocol. They are performed every two hours, while the patient is in prone position, by the nurse who is responsible for his care.

\section{Enteral feeding}

Every critical care nurse knows that enteral feeding is of great importance. So, in a patient lying on his abdomen for 12 or more hours, what happens to the stomach? Does the prone position affect gastric emptying? - Apparently not. In my unit we do not aspirate the stomach before turning the patient. Nor did they in the Berlin ICU we visited. This is supported by a Dutch study, that showed no difference in a patient's ability to empty the stomach, whether in the prone or supine position (van der Voort et al., 1998). All our patients, who receive enteral nutrition, are routinely aspirated every four hours to ensure gastric emptying is occurring. We have also found no difference in the patient's

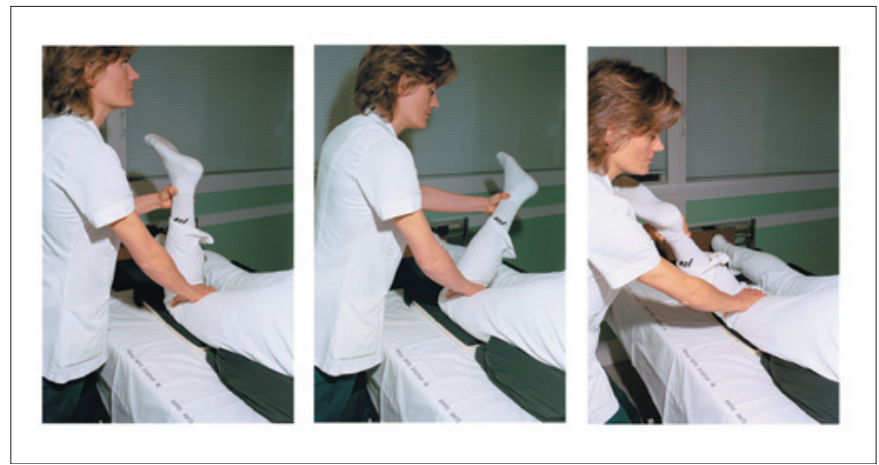

Figure 3.

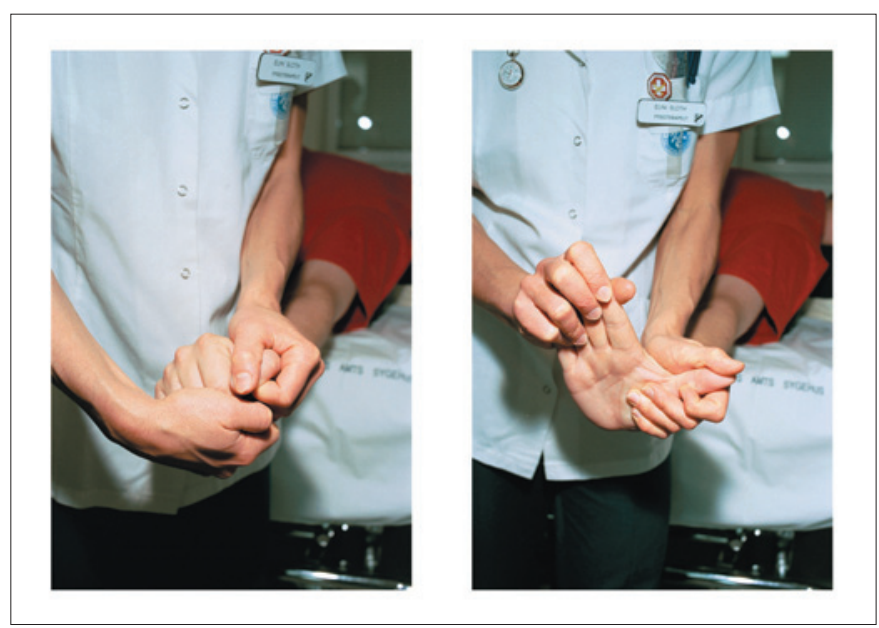

Figure 4.
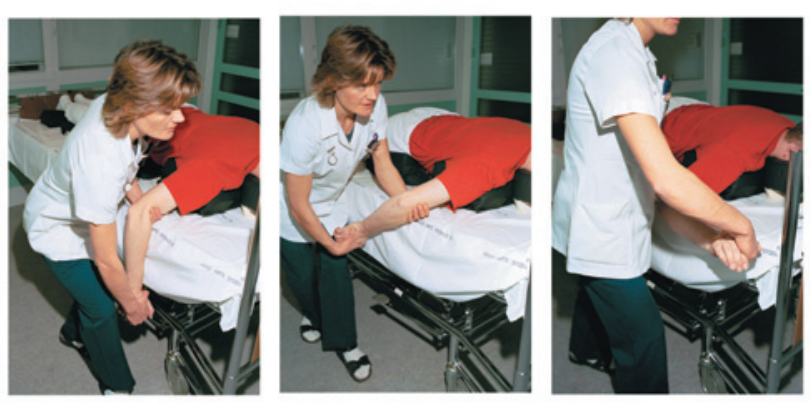

Figure 5. ability to empty the stomach, whether they are in supine or in prone position.

\section{Endotracheal suctioning}

When considering the best way of carrying out endotracheal suctioning of a patient in the prone position, the underlying condition, ARDS, is more important than whether or not the patient is being nursed in the prone position.

Most ARDS patients are ventilated with pressure control and a relatively high level of positive end-expiratory pressure (PEEP) to keep the alveoli open and promote gas exchange. To prevent alveoli collapse, resulting in decreased oxygenation, one must try to avoid loss of PEEP during the suction procedure. This means that the frequency of endotracheal suctioning should be minimised. Nevertheless, it is often necessary to increase the frequency of endotracheal suctioning, when the patient is turned prone, due to the huge large quantity of secretions that occur, when the position is changed.

To minimise the loss of PEEP and airway pressure, we use a bronchoscope swivel connector. This enables the tight passage of a suction catheter with minimal loss of PEEP. Immediately prior to suctioning the patient, we put the ventilator on inspiratory hold (or we clamp the endotracheal tube with forceps immediately after an inspiration), while the catheter is introduced into the proximal end of the tube. As soon as the catheter is inside the tube, we carry out the suctioning procedure.

Manual hyperinflation (or 'bagging') is a debated procedure. Those who recommend bagging do so in the belief that it has a positive effect on compliance and oxygenation (Jones et al., 1992). However, those against the procedure are concerned about overdistension of the alveoli and damage to the lungs (Ciesla, 1996). In my unit, we do not have a set rule, and we decide from patient to patient whether we shall perform the bagging or not, knowing this is not ideal. However, I will leave it to the researchers to develop the evidence for best practice.

In a patient lying with his face pointing directly down into the mattress, it is not easy to perform endotracheal suctioning. In my unit, we have discussed the use of the closed suction system, though at the moment we do not use them. There is no doubt that there are advantages for nurses, but we are not sure of the benefits for patients. As with the bagging procedure, we are searching the literature for the best evidence upon which to base our guidelines.

\section{Care of relatives}

Patients with a severe ARDS, intubated and on a ventilator, are seldom awake. They are usually unconscious or heavily sedated, and so cannot be informed about the turning procedure. Instead, the relatives must be informed. The reaction from the relatives is predominantly positive. More than one relative has said, 'It is almost like seeing him at home. He always sleeps on his stomach!' Facial oedema does not seem to frighten relatives, though we always explain it to them.

\section{Audit}

During the past four years, we have performed approximately 60 prone ventilation turns in approximately 40 patients. We have seen the following complications:

- Two pulmonary artery catheters and two feeding tubes were accidentally pulled out (in four different patients).

- Five patients had pressure sores on the face (forehead, nose or chin).

- One patient developed a pressure sore on his chest. 
- Almost every patient developed oedema in the face, which disappears after a few hours.

Initially, we did not record complications. However, we now evaluate every patient, and every prone period, and we are constantly trying to develop new and better skills with a view to improving outcome.

\section{Preventing the need for intubation and ventilation?}

Most of the patients we turn into the prone position are suffering from ARDS, but we are also turning patients with other oxygenation problems. Two patients with chronic obstructive lung disease (COLD); awake and with no respiratory support apart from a little oxygen, have been turned prone in an attempt to avoid intubation and ventilator treatment. This appeared to be successful. This has inspired us to start experimenting: is it possible to avoid intubation and ventilator treatment by turning a patient with other lung diseases than ARDS into the prone position? If anyone reading this article, has any kind of experience with turning non-intubated and non-ventilated patients into the prone position in an attempt to avoid intubation and ventilatory treatment, please contact me for further discussion.

\section{CONCLUSIONS}

Our experience has shown that prone ventilation, particularly for ARDS, is a very beneficial therapy, often producing positive benefits to oxygenation within a very short space of time. However, it is not without complications. The turning procedure itself is a potentially dangerous time for the patient, but clear guidelines can help us to ensure it is as safe as it can be. With good nursing care, most complications can be avoided.

\section{REFERENCES}

Ashbaugh DG, Bigelow DB, Petty TL. (1967). Acute respiratory distress in adults. Lancet; 2: 319-323.

Cisela ND. (1996). Chest physical therapy for patients in intensive care units. Physical Therapy; 76: 609-625.

Bryan AC. (1974). Comments of devil's advocate. American Review of Respiratory Disease; 110(Suppl): 143-144.

Gustavsson A, Jonsson E. (1998). IVA patient i buklage - en kortlägning om och hur behandlingsmetoden används på IVA-avdelinger i Sverige. (ICU patients in prone position: is it a treatment, and how is it performed in ICUs in Sweden?.) Ventilen; $1: 12-15$. [Danish]

Jones AYM, Hutchinson RC, Oh TE. (1992). Effects on bagging and percussion on total static compliance of the respiratory system. Physiotherapy; 78: 661-665.

Mure M, Martling C-R, Lindahl S. (1997). Dramatic effect on oxygenation in patients with severe acute lung insufficiency treated in the prone position. Critical Care Medicine; 25: 1539-1543.

Stocker R, Neff T, Stein S, Ecknauer E, Trentz O, Russi E. (1997). Prone position and low-volume pressure-limited ventilation improve survival in patients with severe ARDS. Chest; 111: 1008-1017.

van der Voort PHJ, Zandstra DF. (1998). Enteral feeding in critically ill: comparision between supine and prone position. 11th ESICM annual congress - Stockholm 1998 - Physiotherapy/Nursing Abstracts Book. Abstract N17.

Vollman K. (1997). Prone positioning for the ARDS patient. Dimensions of Critical Care Nursing; 16: 184-193.

Willems MCM, Voets AJ, Welten RJTJ. (1998). Two unusual complications of prone-dependency in severe ARDS. Intensive Care Medicine; 24: 276-277. 